\title{
Diagnóstico sobre comprensión conceptual y lectora en estudiantes de la UNAE a través de la "cumbia epistemológica" de Les Luthiers
}

\author{
José Manuel CASTELLANO GIL
}

ORCID iD: http://orcid.org/0000-0002-7044-6564

Universidad Católica de Cuenca, Ecuador. Ecuador

(Recibido el 10 de Marzo de 2020, Aceptado el 25 de Abril de 2020)

RESUMEN: Este estudio surge desde la propia experiencia docente durante siete períodos académicos en la Universidad Nacional de Educación de Ecuador (UNAE), donde hemos observado de forma continuada un grave problema de apropiación conceptual y lectora por parte del estudiantado. De modo que este trabajo intenta acercarse a esa problemática a través de la aplicación de un diagnóstico, además, de evaluar el aprendizaje derivado en un grupo de alumnos de Educación General Básica, que conforman el universo total de estudiantes inscritos en el segundo ciclo de la mencionada carrera.

El encuadre metodológico empleado en este estudio es prioritariamente cuantitativo, a partir de los datos recogidos en un cuestionario principal que se combina con otros instrumentos complementarios. El procedimiento metodológico está sustentado a partir de la utilización de un recurso visual, la cumbia epistemológica del grupo cómico argentino Les Luthiers titulada "Dilema de Amor", al considerar que ese material, dirigido a un público amplio, contiene una serie de elementos referenciales claves, que nos permite indagar en los procesos de comprensión en contexto universitario.

Entre las principales conclusiones obtenidas destacan la existencia de una muy escasa formación de base por parte del alumnado de la UNAE y una actitud o comportamiento generalizado por ocultar o no reconocer las carencias o déficits de conocimiento.

Palabras clave: comprensión lectora; asimilación conceptual; proceso de aprendizaje; UNAE.

\section{Diagnosis of Conceptual and Reading Comprehension in Students de la UNAE Through Les Luthiers' "Epistemological Cumbia"}

\begin{abstract}
This study arises from our own teaching experience during seven academic periods at the National University of Education of Ecuador (UNAE), where we have continuously observed a serious problem of conceptual and reading appropriation by the student body. Therefore, this study attempts to approach this problem through the application of a diagnosis, in addition to evaluating the learning derived from a group of students in General Basic Education, which make up the total universe of students enrolled in the second cycle of the mentioned career.
\end{abstract}


The methodological framework used in this study combines qualitative and quantitative aspects in order to carry out a triangulation between the data collected through a questionnaire, together with the use of other complementary instruments. The methodological procedure is based on the use of a visual resource, the epistemological cumbia of the Argentine comic group Les Luthiers entitled "Dilemma de Amor", considering that this material, aimed at a wide audience, contains a series of key reference elements, which allows us to investigate the processes of understanding in a university context.

Among the main conclusions obtained are the existence of very little basic training on the part of UNAE students and a generalised attitude or behaviour that hides or fails to recognise knowledge deficiencies.

Keywords: Reading Comprehension; Conceptual Assimilation; Learning Process; UNAE.

Correspondencia: E-mail: jmcaste@yahoo.es

\section{Introducción}

La apropiación conceptual junto a la comprensión lectora son elementos claves en todas las etapas formativas del alumnado y de forma especial en la enseñanza superior (Calderón \& Quijano, 2010). No obstante, la amplia brecha existente, entre el conocimiento adquirido por los estudiantes en etapas anteriores (Bonilla et al, 2017) y el nivel de exigencia que debe proponer la formación universitaria (Villegas, 2015), afecta indistintamente a la base de conocimiento y a la formación en habilidades de aprendizaje (López, 2009). Este no es un problema nuevo sino que tiene extensas raíces que provienen de un tiempo pasado y que ha generado una inquietud compartida y generalizada entre el profesorado universitario.

Existen múltiples estudios que han demostrado la existencia de unos bajos índices de práctica y compresión lectora, tanto en el contexto social como en las etapas preuniversitarias y en la enseñanza superior en la región latinoamericana (Soriano \& Alarcón, 2003). Lectura y comprensión son dos habilidades inseparables. De modo que el ejercicio lector no tendrá un efecto sin una comprensión, como tampoco se fomentará una habilidad competencial del aprendizaje y del conocimiento sin una práctica lectora. Ambas carencias, por tanto, merman la capacidad reflexiva, analítica, crítica y comunicativa en el estudiantado.

Nuestra inquietud por este objeto de estudio surge desde la propia experiencia docente en la Universidad Nacional de Educación de Ecuador (UNAE), ubicada en Azogues, Provincia del Cañar. Un centro de reciente creación que cuenta con poco más de cuatro años de existencia. Nacía al amparo del proceso constituyente y del mandato constitucional de 2008, cuya aprobación tendría lugar en 2010. El Proyecto de creación de la UNAE fue plasmado en 2012 (Ministerio de Educación del Ecuador, 2012) y entra en funcionamiento a partir de 2015, bajo un modelo pedagógico basado en problemas, proyectos, casos y en la construcción de conocimientos, actitudes, habilidades, valores, emociones y en su correspondencia entre el campo de la docencia, la investigación, las prácticas preprofesionales y la vinculación a la colectividad (Castellano et al., 2019) y que sería reforzado en 2016 por el Compromiso Presidencial No. 24351 (Ministerio Coordinador de Conocimiento y Talento Humano, 2016). Este centro superior es una de las cuatro universidades denominadas emblemáticas del país, donde gran parte de sus estudiantes eran beneficiarios de un sistema de becas, a los que ya no tienen acceso los nuevos ingresados desde hace dos semestres.

(C) Psy, Soc, \& Educ, 2020, Vol. 12(2) 
Durante siete períodos académicos hemos observado de forma sistemática un grave problema de apropiación conceptual entre los estudiantes. De modo que este trabajo pretende acercarse a esa problemática y realizar un diagnóstico sobre el estado de asimilación conceptual y lectora, además, de evaluar el proceso de aprendizaje derivado en un grupo de alumnos constituido, formado por dos paralelos de segundo ciclo de Educación General Básica que cursaban, durante el primer semestre abril-julio de 2019, la materia de "Investigación acción-participativa: Historia de vida" y otros dos grupos que recibían la asignatura de "Medio Social II" y que vienen a representar al universo total de los alumnos inscritos en el segundo ciclo de la mencionada carrera.

Este diagnóstico pretende obtener y ofrecer una serie de datos sobre los aspectos enunciados, que permita al profesor caracterizar el punto de partida del alumnado, para actuar en el diseño y definición de una estrategia docente, junto a un ejercicio de reflexión profesional, con la idea de contribuir a un proceso de enseñanza-aprendizaje ajustado al perfil y a las características del grupo.

\section{Metodología}

El encuadre metodológico empleado en este estudio es predominantemente cuantitativo, a través de un cuestionario principal, junto a la utilización de otros instrumentos complementarios, como un test sobre conocimientos previos (Castellano et al.. 2018d); prácticas culturales (Castellano et al, 2018a; Castellano et al, 2018c; Loaiza et al. 2018); junto a los aportes cuantitativos obtenidos en los registros de observación directa en el aula; y los auto-relatos de historias de vida del alumnado (Castellano, 2018b).

El procedimiento metodológico está sustentado a partir de la utilización de un recurso visual, la cumbia epistemológica del grupo cómico argentino Les Luthiers titulada "Dilema de Amor" (véase anexo núm. 1) al considerar que ese material, dirigido a un público amplio, contiene una serie de elementos referenciales claves, que nos permite indagar en los procesos de comprensión en contexto universitario. La estructura de este procedimiento fue organizada en cinco fases. La primera consistía en visionar el mencionado vídeo, sin ofrecer información alguna al alumnado. Previo a iniciar la segunda fase se indicaba a los estudiantes que su labor se centraría en registrar todos aquellos léxicos, conceptos y autores contenidos en el vídeo y que desconocían. Para ello se reprodujo el vídeo en dos ocasiones. En la tercera fase, los estudiantes procedían a dar lectura de forma individual y en voz alta sus registros, sin reiterar los señalados anteriormente por sus compañeros, con la finalidad de confeccionar un inventario general. A continuación esa relación fue leída en el aula con la idea de que anotasen los nuevos léxicos, conceptos y autores que por los motivos que fueran no habían sido incluidos en la etapa anterior. La cuarta fase, realizada como ejercicio fuera del salón de clase, tenía como principal tarea la localización del texto o letra de la canción con el propósito de llevar a cabo su lectura; reincorporar nuevos léxicos, conceptos y autores, si fuese el caso, además, de corregir y escribir correctamente las palabras y buscar sus significados. Por último, después de tres semanas, se acometía la quinta y última fase con la aplicación de un ejercicio de verificación del aprendizaje, a través de un instrumento elaborado en Google Forms y estructurado en cinco módulos de contenidos (identificativo; valoración e indagación; evolución de las etapas; y evaluación de la asimilación 
de léxicos, conceptos y autores), que contemplaban un total de 102 ítems, donde se combinaban preguntas abiertas, cerradas junto a otras de opciones múltiples. El cuestionario fue aplicado en el laboratorio de informática de la propia Universidad y cumplimentado durante un tiempo aproximado entre 60 y 90 minutos.

\section{Análisis Y Discusión}

\section{Caracterización de la población objeto de estudio}

Este instrumento se aplicó a los cuatro grupos de estudiantes que cursaban el segundo ciclo de la carrera de Educación Básica en la Universidad Nacional de Educación de Ecuador durante el semestre abril-julio de 2019, correspondiente a la materia "Medio Social II" integrado por 61 alumnos (53\%) y a la asignatura "Investigación Acción-participativa: Historia de vida" con 54 estudiantes $(47 \%)$.

Este universo poblacional está distribuido por un $59,1 \%$ de mujeres y un $40,9 \%$ de varones, con rangos etarios diferentes, por lo que se decidió agruparlos en siete intervalos: entre 19 y 20 años que representa un 29\%; entre 21 y 22 que alcanza un $47 \%$; entre 23 y 24 que asciende a un $16 \%$; entre 25 y 26 años con un $3 \%$; entre 27 y 28 con un 3\%; entre 29 y 30 con un $2 \%$; y más de 30 años con un índice de un 1\%. Esa distribución presenta unas edades elevadas con respecto a la edad natural de los alumnos que deberían cursar su segundo semestre en estudios universitarios.

Con respecto al estado civil de estos estudiantes encuestados, el $92 \%$ son solteros, un $3 \%$ casados, un 3\% en unión libre y un 1\% divorciados. Sin embargo, un 17\% del total cuenta con cargas familiares.

La caracterización socioeconómica de este grupo de estudiantes viene definida por unos bajos ingresos familiares. Así un $87 \%$ se encuentran entre los quintiles más precarios de la sociedad ecuatoriana (un $65 \%$ en el primer quintil y un $22 \%$ en el segundo), mientras que un $9 \%$ en el tercero y un $4 \%$ en el cuarto. Otro dato relevante, en ese sentido, es que para un poco más de la mitad de los alumnos estudiados $(56 \%)$ es la primera generación familiar en ingresar a la formación universitaria.

Un alto porcentaje de estos alumnos (80\%) proceden de instituciones educativas fiscales, es decir públicas, frente a un $14 \%$ de centros fiscomisionales (instituciones privadas cofinanciadas por el Estado) y un 6\% de titularidad jurídica particular o privada.

En cuanto a la procedencia territorial destacan los alumnos originarios de la provincia colindante del Azuay con un 59\% y la del Cañar con un 33\%, que supone un $92 \%$ frente al aporte de otros ámbitos territoriales (Guayas un 3\%; Santiago Morona un 3\%; Loja y Manabí con un 1 respectivamente). Un $67 \%$ de estos universitarios proceden de espacios urbanos, un $43 \%$ del cantón Cuenca, un 24\% del cantón Azogues y con una menor presencia de otras cabeceras cantonales: Paute con un 7\%, Gualaceo y Biblián con un 4,3\% respectivamente, Chordeleg con un $3 \%$, Cañar y El Tambo con 3\% cada uno y Sigsig con un 2\%. Mientras que los ocho cantones restantes (Sevilla de Oro, Deleg, Sucal, Santa Isabel, Morona, Ibarra, Lago Agrio y San Juan Bosco) representan un 1\% respectivamente. De modo que se puede afirmar que la adscripción territorial de este grupo de alumnos de la UNAE se corresponde con los centros urbanos de las 
dos capitales provinciales (Cuenca y Azogues) y con una escasa participación de los cantones agrarios cercanos al centro universitario.

Con respecto a las pruebas de ingreso a la universidad, realizadas por la Secretaría de Educación Superior, Ciencia, Tecnología e Innovación (Senescyt), un 37\% alcanzó la nota de ingreso a la primera vez; un 43\% tuvo que realizar dos pruebas para su ingreso en la educación superior; un $16 \%$ lo obtenía en la tercera ocasión; y un 3\% necesitó de cuatro o más intentos.

Un 25\% de los estudiantes encuestados manifiestan que el puntaje obtenido en la prueba de Senescyt (véase tabla 1) no le permitió cursar su carrera deseada y para un alto porcentaje (37\%) la UNAE no formaba parte como centro universitario preferencial. A ello hay que añadir que un $23 \%$ de estos estudiantes analizados habían desertado de otras universidades y carreras ubicadas en la vecina ciudad de Cuenca.

Tabla 1. Distribución porcentual por intervalos del puntaje obtenido por los alumnos objeto de estudio en la prueba de acceso a la universidad realizado por la Senescyt.

\begin{tabular}{lcc}
\cline { 2 - 3 } & Frecuencia & Porcentaje \\
\hline Entre 700 y 749 & 10 & 8,7 \\
Entre 750 y 799 & 30 & 26,1 \\
Entre 800 y 849 & 35 & 30,4 \\
Entre 850 y 899 & 24 & 20,9 \\
Entre 900 y 949 & 13 & 11,3 \\
Entre 950 y 999 & 3 & 2,6 \\
Total & 115 & 100 \\
\hline
\end{tabular}

\section{Identificación de léxicos, conceptos y autores}

El registro general de palabras desconocidas en las tres fases indica que, sobre los 158 léxicos que contiene la letra de la composición musical empleada en este diagnóstico (excluyendo artículos determinados, conjunciones, conceptos y autores), los estudiantes no llegan a conocer el significado de 47, es decir, un 30\% del total. Mientras que la ausencia de apropiación de conceptos (14) y autores (8) se eleva al 100\%. Un dato altamente significativo en la evolución de los registros en sus distintas fases evidencia una tendencia creciente del número de anotaciones de léxicos, conceptos y autores en las etapas posteriores (ver Tabla 2), que puede entenderse como un comportamiento dirigido a ocultar sus debilidades comprensivas, tanto en la lectura como en el salón de clase. Además se debe señalar que no se aprecian diferencias sustanciales entre los alumnos de ambas materias con respecto a los rangos de palabras registradas durante las tres fases, ni un comportamiento diferenciado entre géneros. 
Tabla 2. Distribución porcentual de léxico, conceptos y autores recogidas en las tres fases

\begin{tabular}{lccc}
\hline Intervalos & Porcentaje $\mathbf{1}^{\mathbf{a}}$ Fase & Porcentaje $\mathbf{2}^{\mathbf{a}}$ Fase & Porcentaje $\mathbf{3}^{\mathbf{a}}$ Fase \\
\hline Entre 1 a 5 & 22,6 & 3,5 & 2,6 \\
Entre 6 a 10 & 59,1 & 10,4 & 6,1 \\
Entre 11 a 15 & 12,2 & 26,1 & 19,1 \\
Entre 16 a 20 & 3,5 & 29,6 & 20,9 \\
Más de 20 & 2,6 & 30,4 & 51,3 \\
Total & 100 & 100 & 100 \\
\hline
\end{tabular}

\section{Resultados del ejercicio de comprensión}

Una vez identificados los problemas de comprensión de léxicos, conceptos y autores en sus distintas fases y tras la labor encomendada a los estudiantes de apropiarse de sus significados y del conocimiento de los autores se incorporó en el diseño del cuestionario una prueba para verificar la asimilación de los mismos. Con respecto al léxico el ejercicio consistía en utilizar ese vocablo en la construcción de una oración. Mientras que en el caso de los conceptos y autores se planteaba una pregunta y se ofrecía la elección de una de las tres respuestas opcionales.

\section{Léxicos}

La cantidad general de léxicos registrados ascendieron a 47, que fueron recogidos en su totalidad en el cuestionario con la finalidad de verificar el proceso de asimilación. Los resultados obtenidos muestran unos datos altamente positivos, donde diez léxicos alcanzan el 100\% de aciertos; veintisiete entre $80 \%$ al $99 \%$; siete entre el $50 \%$ al $79 \%$; y tan sólo tres léxicos se sitúan por debajo del 50\% (véase Figura 1). No obstante, hay que mencionar la presencia de otros elementos negativos asociados, como las excesivas faltas ortográficas, el inadecuado uso léxico, las dificultades de expresión, la precariedad en el desarrollo de ideas y el escaso dominio de contextualización lectora (véase anexo núm. 2). Una realidad que, en cierta manera, podría estar justificada en estos alumnos por encontrarse en un periodo de adaptación a la formación universitaria. Sin embargo, otros datos obtenidos sobre hábitos y prácticas culturales en la UNAE ponen de manifiesto una tendencia general deficitaria y carencias relevantes en los ciclos superiores. Y en ese mismo sentido apunta un informe sobre titulación de grado o Tesis de Licenciatura, donde se resalta las "bajas destrezas de lectura y escritura". 
Diagnóstico sobre comprensión conceptual y lectora...

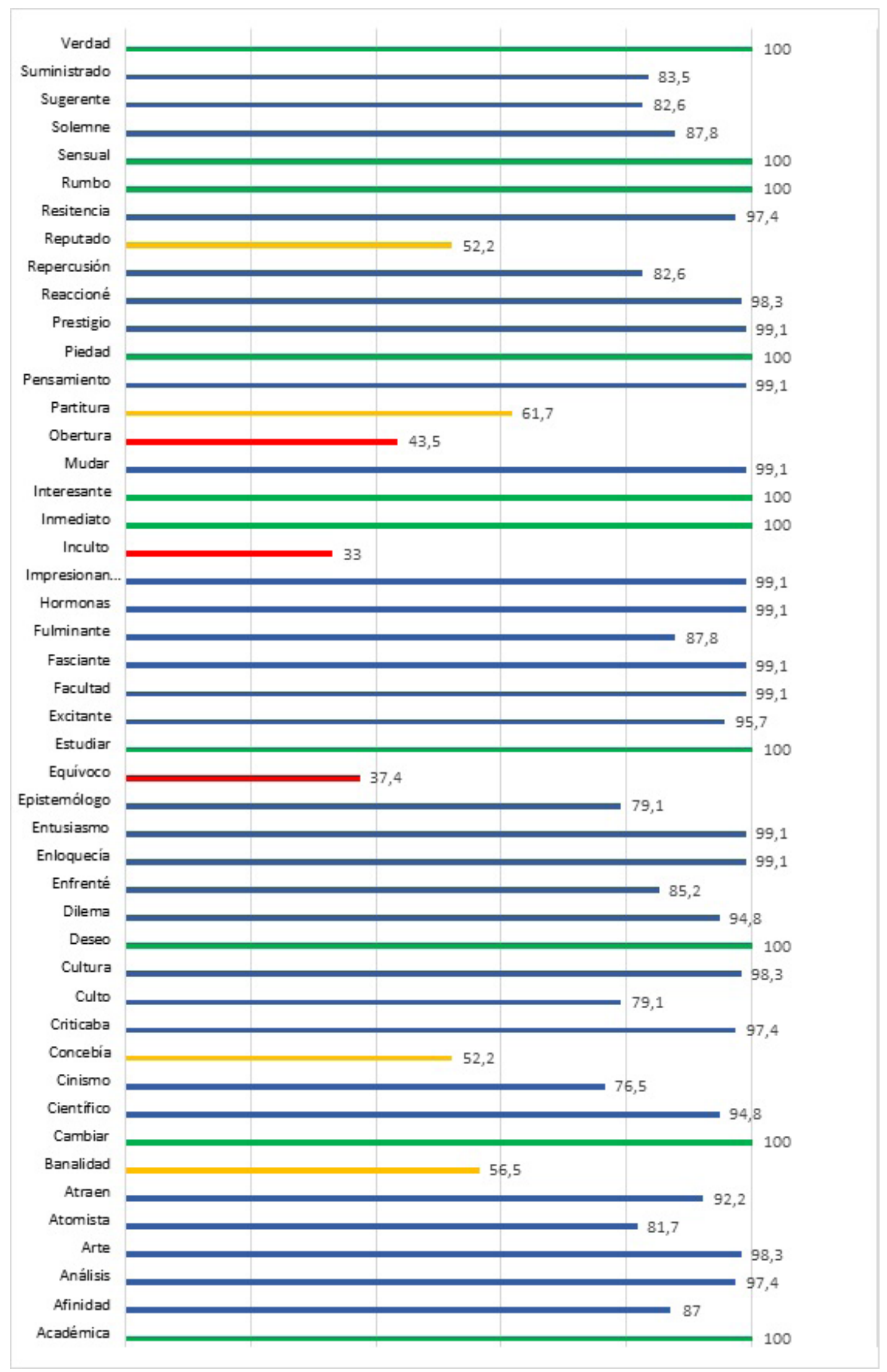

Figura 1. Distribución porcentual de construcción de oraciones correctas a partir del léxico propuesto.

(C) Psy, Soc, \& Educ, 2020, Vol. 12(2) 


\section{Conceptos}

Los resultados en este apartado también ofrecen una valoración muy positiva de aprendizaje, pues diez de los catorce conceptos registrados fueron contestados correctamente entre una franja que oscila entre el 70\% al 94\%; uno (metodología) con un 53\% de acierto; y tres conceptos (idealismo, filosofía y tesis) por debajo del 50\%, como se puede apreciar en la Figura 2.

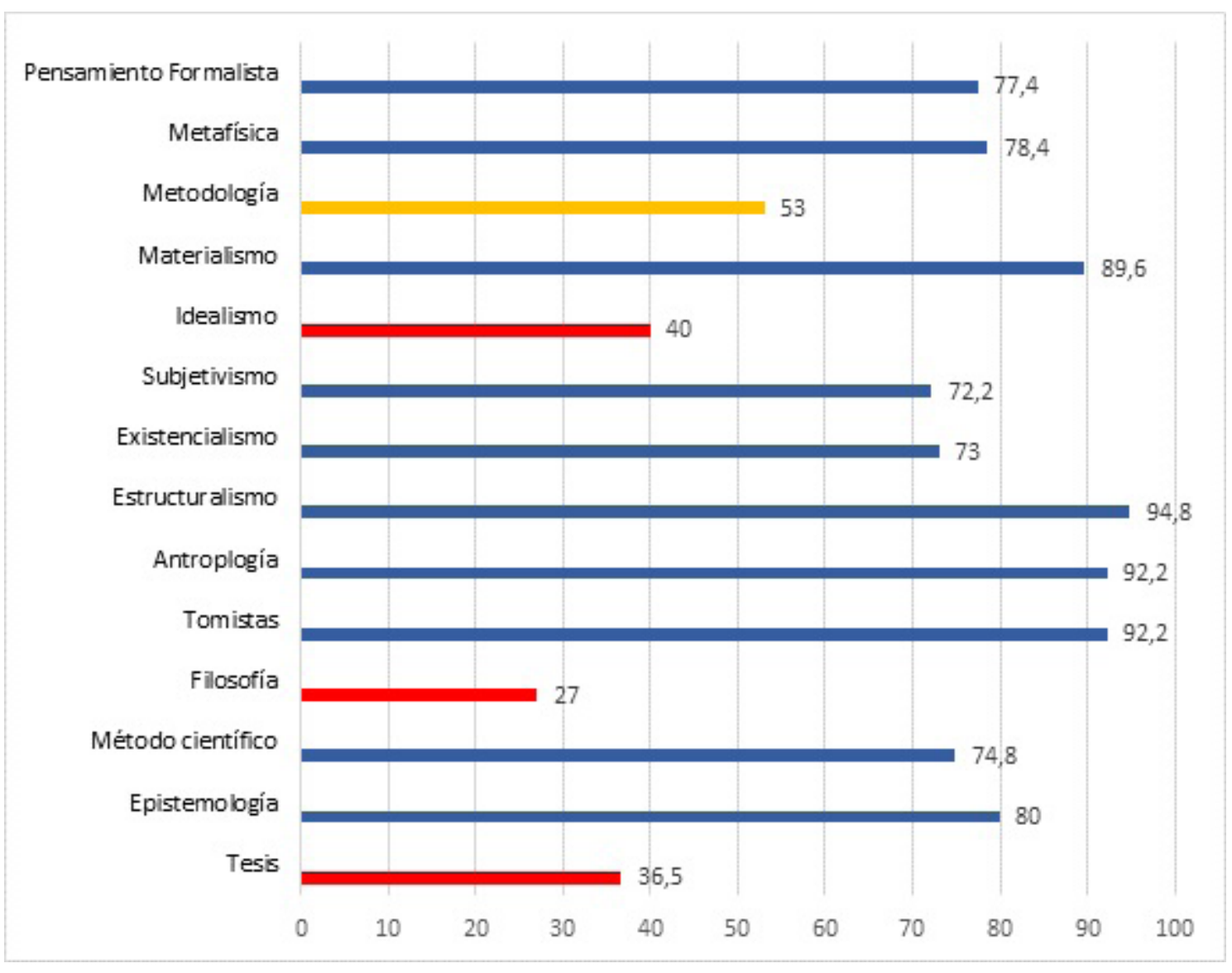

Figura 2. Distribución porcentual de aciertos sobre conceptos indagados bajo la modalidad de tres opciones de respuestas

\section{Autores}

Con respecto al conocimiento sobre los autores referenciados en la letra de la canción se planteó en el cuestionario la siguiente pregunta: ¿Ha oído hablar de estos autores en sus etapas formativas anteriores? Un $41 \%$ señaló que "sí, a algunos de ellos"; un 1\% "sí, a todos"; y un $58 \%$ que "no o nunca lo había oído". De igual manera, los resultados obtenidos confirman un buen proceso de aprendizaje, que se evidencia en que un elevado porcentaje de alumnos, entre un $82 \%$ al $99 \%$, identifica de forma correcta el perfil general de los ocho autores indagados (ver Figura 3). 


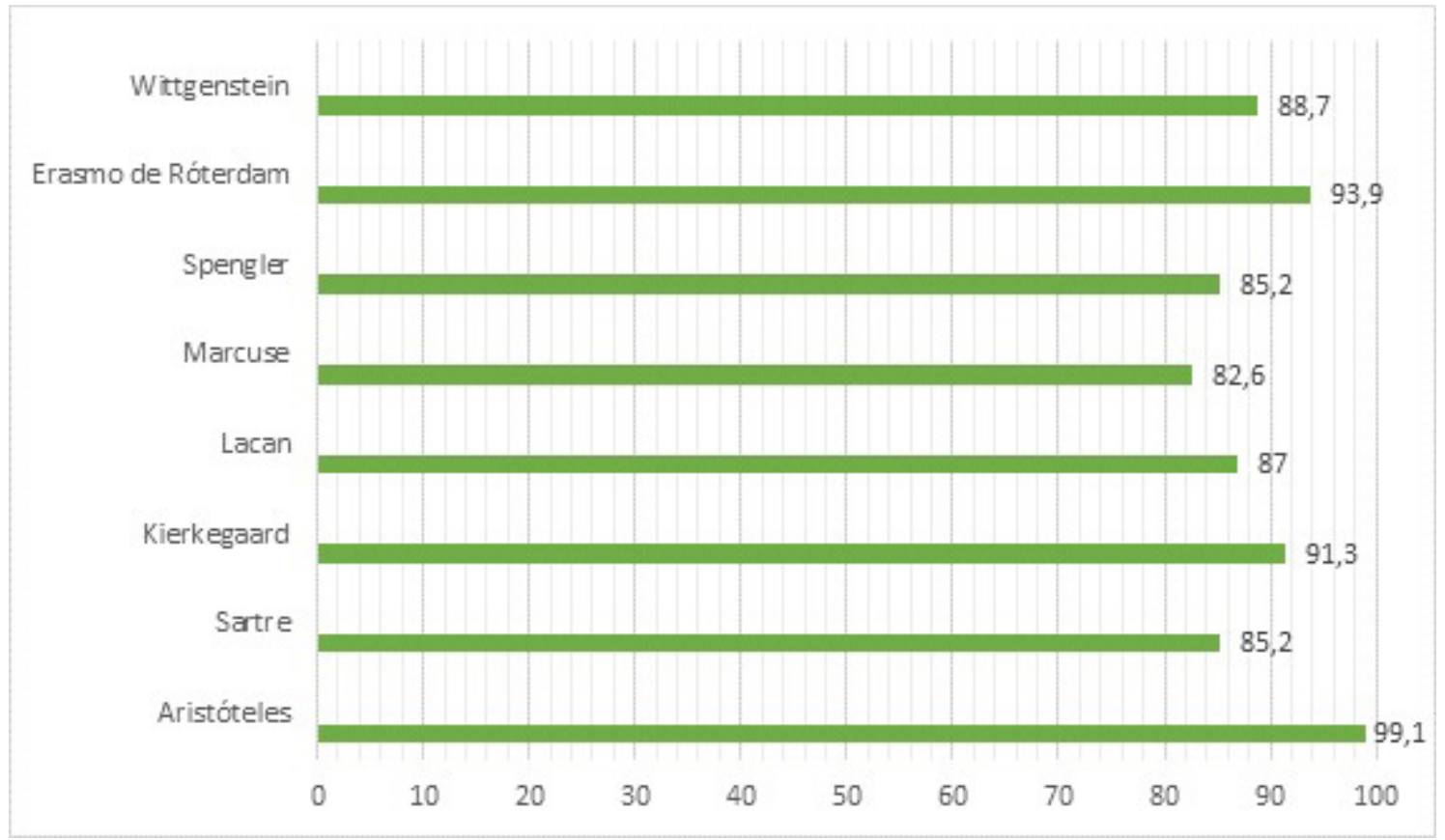

Figura 3. Distribución porcentual de respuestas acertadas con respectos a los autores sondeados

\section{Valoración}

La inmensa mayoría de los estudiantes analizados $(96 \%)$ califica de interesante la prueba realizada con el vídeo del grupo argentino Les Luthiers y un 98\% considera que ese ejercicio les ha dotado de un proceso de aprendizaje significativo. Así para un $69 \%$ de los alumnos esta actividad ha supuesto la apropiación de un nuevo vocabulario y un conocimiento sobre personajes relevantes. Además de ello, a un $8 \%$ le ha permitido comprobar la existencia de otras formas de enseñanza; un 7\% valora la adquisición de conocimientos sobre las corrientes filosóficas y sus representantes; un 5\% señala que se ha apropiado del significado conceptual de epistemología y un $90 \%$ ha declarado que esta tarea realizada ha generado un proceso de reflexión y crítica sobre el sistema educativo ecuatoriano. Y todo ello, a pesar de un contexto general de partida donde el $97 \%$ afirma que no había entendido el vídeo la primera vez que fue visionado, aspecto que también quedó reflejado en los registros de observación directa. Asimismo un $94 \%$ señala que intentaron averiguar posteriormente el significado de las palabras, conceptos y autores que les eran desconocidas en ese momento y que queda constatado a través de los buenos resultados obtenidos en la prueba practicada.

Otro dato altamente relevante es el proporcionado por un $74 \%$ de los universitarios que señala la existencia de dificultades de comprensión de palabras y conceptos durante las clases, aunque un $49 \%$ manifiesta que suele consultar al docente su significado. En cambio, las anotaciones en los registros de observación ponen de relieve un escaso nivel de participación de los estudiantes con respecto a ese comportamiento, con un índice inferior al 3\%. Una tendencia similar se repite con respecto a la comprensión lectora, pues un alto porcentaje (78\%) de estos universitarios confirma la presencia de graves problemas comprensivos en el proceso de lectura, aunque nuevamente un $95 \%$ manifiesta que recurre al uso de diccionarios convencionales o digi- 
tales para resolver esas carencias, pero se debe reiterar que tales acciones tampoco coinciden con los registros de observación directa en relación a las tareas desarrolladas en el aula sobre análisis y comentarios de textos, que apenas alcanzan un $3 \%$.

\section{Conclusiones}

Las principales conclusiones resultantes muestran, en líneas generales, la existencia de una muy escasa formación de base por parte del alumnado de la UNAE y una actitud o comportamiento generalizado por ocultar o no reconocer las carencias o déficits de conocimiento, que se traduce en ese elevado incremento del número de léxicos, conceptos y autores registrados en fases posteriores. Aspecto éste, que también se evidencia cotidianamente en el propio escenario del aula, reflejado en una carencia de comprensión básica, no sólo de conceptos específicos de la materia sino también en el reducido manejo de un soporte léxico elemental, que origina en el alumnado importantes inconvenientes de comprensión y que conlleva al docente a una readaptación del contenido y planificación de la materia. Esta situación podría explicarse tanto por la escasa formación general que arrastran de etapas educativas precedentes como de un comportamiento cultural específico, junto al perfil de ingreso de los estudiantes en la Universidad Nacional de Educación de Ecuador. Esta precariedad, referida en este caso concreto a las corrientes filosóficas, pero que puede extenderse a otras áreas de conocimiento, bien puede responder en gran parte como consecuencia directa del propio diseño curricular de la asignatura de "Filosofía" que se imparte en el Bachillerato General Unificado, que no se detienen en fijar una panorámica general de las corrientes filosóficas y en cuya malla, además, no se prima una formación teórica de contenidos, al igual que el propio diseño curricular de la UNAE, que tampoco potencia el desarrollo de las competencias disciplinarias. De modo que resultaría de gran interés que nuevos estudios, desde diferentes perspectivas y enfoques, ahondaran sobre estas carencias detectadas con la finalidad de contribuir a una reflexión sobre las mismas y que pudieran ser trasladadas a una adaptación curricular con la finalidad de mejorar el proceso de enseñanza-aprendizaje.

\section{Anexo}

\section{Letra de la canción “Dilema de Amor” del grupo Les Luthiers.}

Marcos Mundstock: Otra vez no viene Ramírez... yo creo que, la resistencia del análisis de este hombre tiene que ver con esta tesis sobre Mastropiero, que le han encargado. Me traje unos libros sobre Mastropiero para ver si encuentro alguna pista... por ejemplo, este: "Cultura es todo, incluso Mastropiero"... Capítulo quinto, "Mastropiero y la epistemología": "La epistemología, como todos saben, es la rama de la filosofía que estudia el método científico... Cierta vez le encargaron a Mastropiero una partitura para la solemne entrega del Premio de Epistemología de la Universidad de la Sorbona, en París. Mastropiero aceptó pero, por un equívoco, su obra tuvo más repercusión que la esperada. Le habían encargado una obra para la Sorbona, y Mastropiero entendió 'para las hormonas', y en vez de una obertura académica, compuso una cumbia... una cumbia en la que incluyó algunos nombres de filósofos y epistemólogos, que le habían suministrado con el encargo. Luego de la sorpresa inicial del calificado auditorio, Mastropiero fue reputado de inculto, y de ese modo fue 'reputado' por todos. Este error de Mastropiero le cerró las puertas de 
los círculos filosóficos, pero le abrió un enorme prestigio... en las bailantas. Sobre todo porque esa cumbia, 'Dilema de amor', se convirtió en el gran éxito del popular grupo 'Los Brillantes'..."

\section{Carlos Núñez Cortés:}

Estoy enamorado, por fin me enamoré, el sábado a la noche en el baile la encontré.

Estaba vestida

para enamorar, sensual y atractiva...

Y me miraba sin parar.

Todos: Si dos jóvenes se atraen, ya nada los detiene, ustedes ya saben todo lo que viene.

Carlos Núñez Cortés:

Salimos a bailar, la miré, me miró...

Todos: Y el deseo fulminante... los atrapó.

Carlos Núñez Cortés: Y mientras se movía...

Todos: ¡Qué fascinante!

Carlos Núñez Cortés: Para impresionarme...

Todos: ¡Qué sugerente!

Carlos Núñez Cortés: Se puso a hablar...

Todos: ¡Qué excitante!

Carlos Núñez Cortés: De filosofía...

Todos: Qué interesante...

Carlos Núñez Cortés:

De inmediato reaccioné, y ahí mismo en la pista... 
Daniel Rabinovich: ¿Ahí mismo, en la pista?

\section{Carlos Núñez Cortés:}

...la enfrenté y le pregunté, si era aristotélica o tomista.

Todos: La, la, la, la, la...

Carlos Núñez Cortés:

No paramos de bailar, nuestros labios se atraían

y empezamos a hablar

de epistemología.

Daniel Rabinovich:

Los jóvenes inventan

palabras cada día, se ve que ahora lo llaman...

"Epistemología".

¡Qué bonito, mi amor!

Todos: ¡Qué bonito, mi amor!

Daniel Rabinovich: Hacer cada día...

Todos: Hacer cada día...

Daniel Rabinovich: Juntitos los dos...

Todos: ¡La "Epistemología”!

Carlos Núñez Cortés:

Tocamos muchos temas

de antropología...

Todos: ¡Entonces hicieron la “epistemología”!

Carlos Núñez Cortés: Del estructuralismo, del rumbo del arte y el existencialismo... de Kierkegaard a Sartre.

Daniel Rabinovich: Ella sacudía

Todos: Ella sacudía

(C) Psy, Soc, \& Educ, 2020, Vol. 12(2) 
Daniel Rabinovich: Su estructuralismo

Todos: Su estructuralismo

Daniel Rabinovich: Y él hacía lo mismo...

¡Con su antropología!

Carlos Núñez Cortés:

Y hablando de Marcuse, de Spengler y Lacan

llegamos a Erasmo de Roterdam...

Daniel Rabinovich: Los jóvenes de aman

con tanto entusiasmo,

que sólo con hablar...

¡Ya llegan al «erasmo»!

Todos: Y fueron a la cama

Carlos Núñez Cortés:

No, no, no...

El amor no sólo es

ir con alguien a la cama...

Todos: También se puede hacer...

de pie o en la ventana.

Carlos Núñez Cortés:

Me dijo que leía a Wittgenstein,

Y que la enloquecía

su epistemología...

Daniel Rabinovich:

Yo que tú me cuidaría, ya nombró como a doce, con cualquiera que conoce

hace "epistemología".

Carlos Núñez Cortés:

Yo le dije que ese tipo solo quería

quitar lo metafísico de la filosofía...

Todos: No se puede estar amando metafísico nomás...

Daniel Rabinovich: Ni estar a cada rato... ¡"epistemologando"!

Carlos Núñez Cortés: 
Me preguntó con cinismo

si yo no concebía

otra metodología

que el materialismo.

Todos: Ella criticaba

tu metodología,

pero tipo que veía...

se lo "epistemologaba".

Carlos Núñez Cortés:

¡Le dije que se fuera!

El pensamiento formalista

acaba en idealismo

subjetivo y atomista.

Todos: ¡La mandaste a mudar,

la echaste sin piedad!

Carlos Núñez Cortés:

¡No se debe aceptar

la banalidad!

Ya encontraré a otra

con más afinidad.

Epistemólogas hay muchas

en la facultad.

Todos: Nos has revelado

otra realidad,

nos has señalado...

¡el camino a la verdad!

Daniel Rabinovich: Vamos a cambiar

Todos: Vamos a cambiar

Daniel Rabinovich: Esta vida vacía...

Todos: Esta vida vacía...

Vamos todos a estudiar...

¡»Epistemología»! 


\section{Breve selección de oraciones construidas por los alumnos a partir del léxico propuesto (en mayúsculas). Se ha respetado fielmente en esta transcripción la ortografía original.}

1. El profesor CONCEBÍA permisos.

2. Pablo CONCEBIO ese dia.

3. La persona que no hacen daño son personas CULTAS.

4. Ella tiene una casa ENFRENTÉ de un hospital.

5. Los estudiantes estudian matemáticas ya que es EQUÍVOCO.

6. EXCITANTE es un palabra que define a una persona segun su estado en que se encuentre.

7. Por AFINIDAD, María y Pedro son los padres de la niña.

8. Al escribir un texto debe escribir con ANALISIS a las ideas que se escriben.

9. En mi bario existe una BANALIDAD de pandillas

10. La educación tradicional es una BANALIDAD con los alumnos.

11. Algunos educadores poseen CINISMO ante sus estudiantes, es decir son doble personalidad.

12. Lucia cometio un PRESTIGIO de mentiras.

13. Antes de entrar a la universidad, REACCIONÉ sobre la carrera que quería seguir.

14. Mediante la REPERCUSION se comprende mas.

15. Una persona que sienta REPERCUSION por expresar sus ideas no puede sentirse libre.

16. La REPERCUSIÓN es muy mala en la sociedad.

17. La policía realizó un trabajo de REPERCUSIÓN.

18. El REPUTADO es de gran influencia en los juzgados ya que es muy prestigioso.

19. El REPUTADO es malo para las personas.

20. El REPUTADO obtuvo consecuencias en su dialogo.

21. Mi primo por estar con amistades que no debe cometió un grave error, ahora es un REPUTADO entre la familia.

22. Fue un partido muy REPUTADO.

23. Mi hermano subio de puesto a SUGERENTE.

24. Un alumno SUMINISTRADO es quien solo memoriza conceptos, pero no los emprende.

25. Fui SUMINISTRADO hoy en la escuela.

26. A mi tio en el trabajo lo clasifican como SUMINISTRADO.

27. El amor SUMINISTRADO no funciona en esta época.

28. El niño esta SUMINISTRADO por causa del sistema educativo.

29. Siempre sera un EQUÍVOCO sin remedio.

30. La actitud es pesima de una persona EQUÍVICO.

31. Todos estan EQUÍVOCO por no saber leer.

32. Piaget en sus estudios fue un ser FULMINANTE.

33. Lo FULMINANTE puede resultar aveces muy malo.

34. No tubo mucho INCULTO el festival.

35. El grupo de personas son INCULTAS no respetan la religion de cada persona.

36. Si no recoges la basura seras INCULTO.

37. Es necesario que mi impaciencia se tenga que MUDAR.

38. La OBERTURA de ese instrumento es muy interesante.

39. Se alejo de la OBERTURA del pozo porque corria mucho peligro.

40. En el río hay una OBERTURA que permite pasar basura.

41. La OBERTURA de la sociedad esta en la situacion en la que esta.

42. En la carretera hay una OBERTURA. 
43. Al cabo de los instantes ajustó una OBERTURA para aumentar el flujo del aire en la cabina.

44. La OBERTURA del docente es grandiosa al comenzar la clase.

45. En la iglesia de Roma en lo mas profundo existen PARTITURAS de la vida de los padres.

46. La PARTITURA de su casa esta mal.

47. El temblor del domingo dejo una PARTITURA en mi hogar.

48. Todos tienen una PARTITURA de miedo.

49. El autor tenia PRESTIGIO dentro de la obra expuesta.

50. Mia no tiene RESISTENCIA por juan.

51. Una persona que es SOLEMNE cuida y acompaña.

\section{Referencias bibliográficas}

Bonilla Marchán, A., Delgado, R. \& Stefos, E. (2017). The Social Characteristics of Postgraduate Students in Ecuador: A Multidimensional Statistical Analysis. Review of European Studies, Vol. 9, No. 2; 2017. pp. 35-44. doi:10.5539/res.v9n2p35

Calderón Ibáñez, A. \& Quijano Peñuela, J. (2010). Características de comprensión lectora en estudiantes universitarios. Revista Estudios Socio-Jurídicos, vol. 12, núm. 1, enero-junio, 2010, pp. 337-364 Universidad del Rosario Bogotá, Colombia. Disponible en https://www. redalyc.org/pdf/733/73313677015.pdf

Castellano Gil, J. M.; Stefos, Efstathios \& Fajardo, A. (2019). Implementación de modelos pedagógicos en Ecuador: un estudio de caso. Revista de Historia, Patrimonio, Arqueología y Antropología Americana. Año 2019, No. 1, Julio (88-116).

Castellano, J. M, \& Reiban, D. (2018a). Indicadores culturales en el contexto universitario: un caso de estudio en la Universidad Nacional de Educación de Ecuador. Revista Chakiñan de Ciencias Sociales y Humanidades, (5), 102-118. Recuperado de http://scielo.senescyt.gob. ec/scielo.php?script=sci_arttext\&pid=S2550-67222018000100102\&lng=pt\&nrm $=$ iso

Castellano, J. M. (coord.). (2018b). Aproximación a la educación ecuatoriana (2000-2018): Relatos de Historia de la vida en el contexto educativo por estudiantes universitarios. Azogues: Casa de la Cultura Ecuatoriana Benjamín Carrión, Núcleo del Cañar.

Castellano, J. M., Fajardo, A. \& Loaiza, K. (2018c). Aproximación al consumo, hábitos y prácticas culturales en la Universidad Nacional de Educación de Ecuador. VII Congreso Internacional de Educación y Aprendizaje, París.

Castellano, J. M., Stefos, E., Sánchez M., Torres, K. \& Reiban, D. (2018d). Un caso de estudio sobre conocimiento previo en tres universidades ecuatorianas: UC, UDA y UNAE. Recuperado de https://scielo.conicyt.cl/scielo.php?pid=S0718-07052018000100377\&script=sci arttext\&tlng $=\mathrm{p}$

Loaiza, K., Fajardo, A. \& Castellano, J. M. (2018). Equipamientos, hábitos y uso de las nuevas tecnologías en estudiantes universitarios: el caso de la UNAE (Ecuador). Congreso de Riobamba.

López Recacha, J. A. (2009). La importancia de los conocimientos previos para el aprendizaje de nuevos contenidos. Disponible en: http://www.csi-csif.es/andalucia/modules/mod_ense/ revista/pdf/Numero_16/JOSE\%20ANTONIO_LOPEZ_1.pdf 
Ministerio Coordinador de Conocimiento y Talento Humano (2016). Compromiso Presidencial "Poner los objetivos de todas las universidades por escrito. Planificación de entrada y salida de profesores. Planificación a 20 años".

Ministerio de Educación del Ecuador Subsecretaría de Desarrollo Profesional Educativo (2012). Proyecto: Creación de la Universidad Nacional de Educación en Azogues, Cañar. Ministerio de Educación del Ecuador Subsecretaría de Desarrollo Profesional Educativo.

Villegas, D. J. M., \& Pereira, R. E. V. (2015). Papel de los conocimientos previos en el aprendizaje de la matemática universitaria. Acta Scientiarum. Education, 37(1), 85. Disponible en: http://search.proquest.com/openview/dcb3b4ec170857dfefc06e147cc36a81/1?pqorigsite $=$ gscholar $\& \mathrm{cbl}=2037656$ 